\title{
PROPER BASES AND AUTOMORPHISMS IN THE SPACE OF ENTIRE FUNCTIONS ${ }^{1}$
}

\author{
MAYNARD G. ARSOVE
}

1. Introduction. We shall be concerned here with the linear space $\Gamma$ of all entire functions, topologized by the metric of uniform convergence on compact sets. This space has been studied in detail by V. Ganapathy Iyer $[2 ; 3 ; 4 ; 5]$, using the equivalent metric $N(f-g)$, where $N(f)$ is defined in terms of the Taylor coefficients $a_{n}$ of $f$ as

$$
N(f)=\sup \left[\left|a_{0}\right|,\left|a_{n}\right|^{1 / n}(n \geq 1)\right] .
$$

A basis in $\Gamma$ is a sequence ${ }^{2}\left\{\alpha_{n}\right\}$ of entire functions such that every entire function $f$ can be represented as

$$
f=\sum_{n=0}^{\infty} c_{n} \alpha_{n}
$$

$\left\{c_{n}\right\}$ being a sequence of complex numbers determined uniquely by $f$ and convergence being interpreted according to the topology on $\Gamma$.

Power series expansions involve the basis functions

$$
\delta_{n}(z)=z^{n},
$$

and the basis $\left\{\delta_{n}\right\}$ has the property that if $f=\sum_{n=0}^{\infty} a_{n} \delta_{n}$ then $\left|a_{n}\right|^{1 / n} \rightarrow 0$. With this as motivation Ganapathy Iyer [4] has called a basis $\left\{\alpha_{n}\right\}$ "proper" provided the coefficients $c_{n}$ in the expansion (1.1) of an arbitrary entire function $f$ satisfy

$$
\left|c_{n}\right|^{1 / n} \rightarrow 0 \text {. }
$$

The conjecture of Ganapathy Iyer that all bases are proper is seen to be false, since the possibility of scaling a proper basis by a coefficient sequence seems not to have been ruled out. For example, $\alpha_{n}(z)$ $=z^{n} / n$ ! defines a basis in which the expansion of the exponential function is given by $c_{n}=1(n=0,1, \cdots)$.

In the present paper the designation proper is reserved for bases whose behavior more closely parallels that of $\left\{\delta_{n}\right\}$. It turns out that for our purposes the important properties to be abstracted from $\left\{\delta_{n}\right\}$ relate to uniform absolute convergence of the expansions (1.1) and to

Presented to the Society, November 12, 1955; received by the editors January 27, 1956 and, in revised form, June 26, 1956.

1 The research reported upon here was supported in part by the National Science Foundation.

2 Throughout the paper the sequences which arise will be indexed by $n=0,1,2, \cdots$ (or by $k=0,1,2, \cdots)$. 
growth of the coefficient sequences $\left\{c_{n}\right\}$. This will be made precise later in Definition 1, but we remark here that our growth rate restrictions rule out the basis defined by $\alpha_{n}(z)=n ! z^{n}$, which is proper in the sense of Ganapathy Iyer. ${ }^{3}$

Moreover, while it is usual to state the convergence properties of power series in terms of uniform and absolute convergence, the proofs actually yield uniform convergence for the series of absolute values. This property, not generally needed in dealing with power series, plays a crucial role in the theory of proper bases.

Using our modified notion of proper basis, we obtain a characterization of such bases in terms of the maximum of $\left|\alpha_{n}(z)\right|$ on $|z|=R$. This in turn leads to a characterization of the linearly homeomorphic images of $\Gamma$ in itself as those closed subspaces admitting proper bases. In particular, we settle a point left unresolved by Ganapathy Iyer $[4$, p. 882] by showing that the proper bases in $\Gamma$ are exactly those sequences which map into $\left\{\delta_{n}\right\}$ under automorphisms of $\Gamma$.

It is a pleasure to acknowledge here many interesting and fruitful discussions with Professor Arthur E. Livingston on subjects relating to the present paper.

2. Preliminaries. Inasmuch as the linear combinations on which we focus our attention are infinite series, the linear space terminology to be employed represents a departure from conventional terminology. We begin, therefore, by listing a few definitions.

Let $\left\{\alpha_{n}\right\}$ be a sequence of functions in $\Gamma$. If $\sum_{n=0}^{\infty} c_{n} \alpha_{n}=0$ implies $c_{n}=0(n=0,1, \cdots)$ for all sequences $\left\{c_{n}\right\}$ of complex numbers for which the series converges uniformly on compact sets, the sequence $\left\{\alpha_{n}\right\}$ will be called linearly independent (otherwise, linearly dependent). We shall say that $\left\{\alpha_{n}\right\}$ spans a subspace $\Gamma_{0}$ of $\Gamma$ provided $\Gamma_{0}$ consists of all linear combinations $\sum_{n=0}^{\infty} c_{n} \alpha_{n}$, where $\left\{c_{n}\right\}$ is any sequence of complex numbers for which the series converges uniformly on compact sets. A sequence $\left\{\alpha_{n}\right\}$ which is linearly independent and spans a subspace $\Gamma_{0}$ will be said to be a basis in $\Gamma_{0}$.

When the condition that $\sum_{n=0}^{\infty}\left|c_{n} \alpha_{n}\right|$ converge uniformly on compact sets is adjoined to those of the preceding definitions, we shall qualify the nomenclature by inserting the word absolute. Thus, $\left\{\alpha_{n}\right\}$ is absolutely linearly independent if $\sum_{n=0}^{\infty} c_{n} \alpha_{n}=0$ implies $c_{n}=0$ $(n=0,1, \cdots)$ for all sequences $\left\{c_{n}\right\}$ of complex numbers for which $\sum_{n=0}^{\infty}\left|c_{n} \alpha_{n}\right|$ converges uniformly on compact sets. Similarly, if $\left\{\alpha_{n}\right\}$

3 This basis furnishes a counterexample to the conjecture of Ganapathy Iyer that for all bases $\left\{\alpha_{n}\right\}$ proper in his sense the sequence $\left\{\left|\alpha_{n} ; R\right|^{1 / n}\right\}$ (see $\S 2$ ) is bounded for each $R>0$. 
is absolutely linearly independent and absolutely spans a subspace $\Gamma_{0}$, it will be termed an absolute basis in $\Gamma_{0}{ }^{4}$

We come now to the definition of a proper basis.

Definition 1. A sequence $\left\{\alpha_{n}\right\}$ of entire functions will be said to be a proper basis for a subspace $\Gamma_{0}$ of $\Gamma$ provided

(1) $\left\{\alpha_{n}\right\}$ is an absolute basis in $\Gamma_{0}$ and

(2) for every sequence $\left\{c_{n}\right\}$ of complex numbers the series $\sum_{n=0}^{\infty}\left|c_{n} \alpha_{n}\right|$ converges uniformly on compact sets if and only if $\left|c_{n}\right|^{1 / n} \rightarrow 0$.

The basis $\left\{\delta_{n}\right\}$ is, of course, trivially proper. It has the further property that if $\sum_{n=0}^{\infty} c_{n} \delta_{n}$ converges uniformly on compact sets, then so does the corresponding series of absolute values. As will be seen later (Corollary 4.1), this property extends to all proper bases which span closed subspaces of $\Gamma$.

Given a sequence $\left\{\alpha_{n}\right\}$ of entire functions, we set

$$
M_{n}(R)=\max _{|z|=R}\left|\alpha_{n}(z)\right|
$$

and employ the notation of Ganapathy Iyer:

$$
\left|\alpha_{n} ; R\right|=\sum_{k=0}^{\infty}\left|A_{n k}\right| R^{k}
$$

where

$$
\alpha_{n}(z)=\sum_{k=0}^{\infty} A_{n k} z^{k}
$$

From the Cauchy inequality $\left|A_{n k}\right| \leq M_{n}(2 R) /(2 R)^{k}$ we obtain at once

$$
M_{n}(R) \leq\left|\alpha_{n} ; R\right| \leq 2 M_{n}(2 R),
$$

a result which allows the interchange of $M_{n}(R)$ and $\left|\alpha_{n} ; R\right|$ in much of the following work.

3. A characterization of proper bases. Although the basis functions $\delta_{n}$ are all polynomials (hence of order zero), it is clear from the outset that proper bases cannot be characterized by the growth properties of the individual basis functions $\alpha_{n}$. In fact, if $\rho$ is any positive integer or $+\infty$, an appropriate choice of $\phi$ in the expression

$$
\alpha_{n}(z)=z^{n} \exp [\phi(z)]
$$

yields a proper basis in which all $\alpha_{n}$ have order $\rho$.

4 This corresponds to the terminology "absolutely convergent basis" as used by Karlin [6]. 
On the other hand, a study of the basis functions $\alpha_{n}$ from the point of view of their growth in $n$ reveals that certain aspects of this growth, manifest in the case of $\left\{\delta_{n}\right\}$, are typical of proper bases in general.

Theorem 1. Let $\left\{\alpha_{n}\right\}$ be an absolute basis for a subspace $\Gamma_{0}$ of $\Gamma$. Then $\left\{\alpha_{n}\right\}$ is proper if and only if

$$
\limsup _{n \rightarrow \infty}\left[M_{n}(R)\right]^{1 / n}<+\infty \quad \text { for each } R>0
$$

and

$$
\lim _{R \rightarrow \infty}\left\{\liminf _{n \rightarrow \infty}\left[M_{n}(R)\right]^{1 / n}\right\}=+\infty .
$$

From (2.1) it is apparent that Theorem 1 remains valid when $M_{n}(R)$ is replaced by $\left|\alpha_{n} ; R\right|$, but the proof is more conveniently carried out in terms of $M_{n}(R)$. What we actually do is to prove two theorems which, when combined, form Theorem 1. First, however, we state the elementary

LEMMA 1. Let $\left\{\phi_{n}\right\}$ be a sequence of entire functions such that $\sum_{n=0}^{\infty}\left|\phi_{n}\right|$ converges to a function bounded on compact sets. Then given $\lambda>1$ and $R>0$, there is an integer $p$ for which $n>p$ implies

$$
\max _{|z|=R}\left|\phi_{n}(z)\right|^{1 / n}<\lambda .
$$

For condition (1) of Theorem 1 we have

THEOREM 2. Let $\left\{\alpha_{n}\right\}$ be a sequence of entire functions. Then the condition

$$
\limsup _{n \rightarrow \infty}\left[M_{n}(R)\right]^{1 / n}<+\infty \quad(\text { all } R>0)
$$

is necessary and sufficient that for all sequences $\left\{c_{n}\right\}$ of complex numbers such that $\left|c_{n}\right|^{1 / n} \rightarrow 0$ the series $\sum_{n=0}^{\infty}\left|c_{n} \alpha_{n}\right|$ converges to a function bounded on compact sets. Moreover, when this condition holds, $\sum_{n=0}^{\infty}\left|c_{n} \alpha_{n}\right|$ converges uniformly on compact sets, and $\sum_{n=0}^{\infty} c_{n} \alpha_{n}$ is an entire function.

Proof. (Sufficiency). For each $R>0$ there is a constant $M(R)$ such that $\left[M_{n}(R)\right]^{1 / n} \leq M(R)$ for all $n$. The conclusion then follows from the fact that for $|z| \leq R$ the series $\sum_{n=0}^{\infty}\left|c_{n} \alpha_{n}(z)\right|$ is dominated by the convergent series $\sum_{n=0}^{\infty}\left|c_{n}\right|[M(R)]^{n}$.

(Necessity). Let us suppose the assertion false, so that the given hypothesis is satisfied but for some $R>0$ the sequence $\left\{\left[M_{n}(R)\right]^{1 / n}\right\}$ is unbounded. Then there exists an increasing sequence $\left\{n_{k}\right\}$ of positive integers such that 


$$
\left[M_{n_{k}}(R)\right]^{1 / n_{k}}>k,
$$

and the sequence $\left\{c_{n}\right\}$ defined by

$$
\begin{aligned}
c_{n_{k}} & =2^{n_{k}} / M_{n_{k}}(R), \\
c_{n} & =0
\end{aligned}
$$

has the property that $\left|c_{n}\right|^{1 / n} \rightarrow 0$. However,

$$
\max _{|z|=R}\left|c_{n_{k}} \alpha_{n_{k}}(z)\right|^{1 / n_{k}}=2,
$$

contradicting Lemma 1 . It follows that $\left\{\left[M_{n}(R)\right]^{1 / n}\right\}$ must be bounded for all $R$, and the proof is complete.

Condition (2) of Theorem 1 is covered by the following theorem.

THEOREM 3. Let $\left\{\alpha_{n}\right\}$ be a sequence of entire functions. Then the condition

$$
\lim _{R \rightarrow \infty}\left\{\liminf _{n \rightarrow \infty}\left[M_{n}(R)\right]^{1 / n}\right\}=+\infty
$$

is necessary and sufficient that $\left|c_{n}\right|^{1 / n} \rightarrow 0$ for all sequences $\left\{c_{n}\right\}$ of complex numbers such that $\sum_{n=0}^{\infty}\left|c_{n} \alpha_{n}\right|$ converges to a function bounded on compact sets.

Proof. (Sufficiency). Let $\left\{c_{n}\right\}$ be a sequence of complex numbers for which $\sum_{n=0}^{\infty}\left|c_{n} \alpha_{n}\right|$ converges to a function bounded on compact sets, and assume that $\lim \sup _{n \rightarrow \infty}\left|c_{n}\right|^{1 / n}>\epsilon>0$. There then exists an increasing sequence $\left\{n_{k}\right\}$ of positive integers such that

$$
\left|c_{n_{k}}\right|^{1 / n_{k}}>\epsilon .
$$

By hypothesis we can find an $R>0$ yielding

$$
\underset{n \rightarrow \infty}{\lim \inf }\left[M_{n}(R)\right]^{1 / n}>2 / \epsilon .
$$

Hence, $\left[M_{n}(R)\right]^{1 / n}>2 / \epsilon$ holds for all sufficiently large values of $n$, so that ultimately

$$
\left|c_{n_{k}} M_{n_{k}}(R)\right| 1 / n_{k}>2 .
$$

Since this contradicts Lemma 1 , we conclude that $\left|c_{n}\right|^{1 / n} \rightarrow 0$.

(Necessity). Assuming that

$$
\lim _{R \rightarrow \infty}\left\{\liminf _{n \rightarrow \infty}\left[M_{n}(R)\right]^{1 / n}\right\}<M<+\infty,
$$

we infer from the monotoneity of the maximum functions that for each $R>0$ 


$$
\lim _{n \rightarrow \infty} \inf \left[M_{n}(R)\right]^{1 / n}<M .
$$

There thus exists an increasing sequence $\left\{n_{k}\right\}$ of positive integers such that

$$
\left[M_{n_{k}}(k)\right]^{1 / n_{k}}<M .
$$

Hence, given any $R>0$, we have

$$
\left|\alpha_{n_{k}}(z)\right|<M^{n_{k}}
$$

for $|z| \leq R$ and $k>R$. It follows that if we define $\left\{c_{n}\right\}$ by

$$
\begin{array}{rlrl}
c_{n_{k}} & =(2 M)^{-n_{k},} \\
c_{n} & =0 & \text { for } n \neq n_{k},
\end{array}
$$

then $\sum_{n=0}^{\infty}\left|c_{n} \alpha_{n}\right|$ converges to a function bounded on compact sets, but $\lim \sup _{n \rightarrow \infty}\left|c_{n}\right|^{1 / n}=1 / 2 M$. This contradiction completes the proof.

Together Theorems 2 and 3 yield Theorem 1 and, in fact, a slightly more general result, since we have nowhere made use of the absolute linear independence of $\left\{\alpha_{n}\right\}$.

In $\$ 1$ we indicated how a proper basis may be scaled by a coefficient sequence to provide an improper absolute basis. From Theorem 1 it is evident that improper absolute bases can also be constructed by rearranging proper bases. For example, we can easily rearrange $\left\{\delta_{n}\right\}$ to obtain a basis $\left\{\alpha_{n}\right\}$ in which $\alpha_{2 k}=\delta_{2 k}(k=0,1, \cdots)$, and for this absolute basis condition (2) of Theorem 1 fails.

4. Linear homeomorphisms and automorphisms. With the aid of the inequalities (2.1), Theorem 6 of [4] can be transcribed as follows: let $\left\{\alpha_{n}\right\}$ be a sequence of entire functions; then a necessary and sufficient condition that there exist a continuous linear mapping $T$ of $\Gamma$ into itself such that $T \delta_{n}=\alpha_{n}(n=0,1, \cdots)$ is that

$$
\limsup _{n \rightarrow \infty}\left[M_{n}(R)\right]^{1 / n}<+\infty \quad(\text { all } R>0) \text {. }
$$

Furthermore, it is readily seen that this implies that if $T$ is given as a linear transformation of $\Gamma$ into itself and $\left\{\alpha_{n}\right\}$ is defined by $\alpha_{n}=T \delta_{n}$, then condition (4.1) is necessary and sufficient for $T$ to be continuous.

In conjunction with Theorem 1 this gives rise to a characterization of the linearly homeomorphic images of $\Gamma$ in itself as the closed subspaces admitting proper bases.

THEOREM 4. If $T$ is a linear homeomorphic mapping of $\Gamma$ into itself, then $\left\{T \delta_{n}\right\}$ is a proper basis in some closed subspace $\Gamma_{0}$ of $\Gamma$. Conversely, 
if $\left\{\alpha_{n}\right\}$ is a proper basis in a closed subspace $\Gamma_{0}$ of $\Gamma$, then there exists a linear homeomorphic mapping $T$ of $\Gamma$ onto $\Gamma_{0}$ such that $T \delta_{n}=\alpha_{n}$ $(n=0,1, \cdots)$.

Proof. Let $T$ be a linear homeomorphism of $\Gamma$ into itself. Then obviously its range $\Gamma_{0}$ is a closed subspace, and we proceed to show that the sequence $\left\{\alpha_{n}\right\}$ defined by $\alpha_{n}=T \delta_{n}$ is a proper basis in $\Gamma_{0}$. Suppose that $f$ is any function in $\Gamma_{0}$, so that $T^{-1} f=\sum_{n=0}^{\infty} c_{n} \delta_{n}$, where $\left|c_{n}\right|^{1 / n \rightarrow 0}$. By the linearity and continuity of $T$ there results $f$ $=\sum_{n=0}^{\infty} c_{n} \alpha_{n}$, and, since (4.1) holds, $\sum_{n=0}^{\infty}\left|c_{n} \alpha_{n}\right|$ converges uniformly on compact sets. Hence, $\left\{\alpha_{n}\right\}$ spans $\Gamma_{0}$ absolutely. That $\left\{\alpha_{n}\right\}$ is a proper basis in $\Gamma_{0}$ is then apparent from the fact that $f$ and $T^{-1} f$ have the same coefficient sequences relative to $\left\{\alpha_{n}\right\}$ and $\left\{\delta_{n}\right\}$, respectively.

For the converse we start with a closed subspace $\Gamma_{0}$ admitting a proper basis $\left\{\alpha_{n}\right\}$ and apply (4.1) to obtain a continuous linear mapping $T$ of $\Gamma$ into itself, carrying $\delta_{n}$ into $\alpha_{n}(n=0,1, \cdots)$. Taking $\phi$ as any function in $\Gamma$, so that $\phi=\sum_{n=0}^{\infty} c_{n} \delta_{n}$ with $\left|c_{n}\right|^{1 / n} \rightarrow 0$, we again infer from the linearity and continuity of $T$ that $T \phi=\sum_{n=0}^{\infty} c_{n} \alpha_{n}$. It follows that $T$ is one-to-one and maps $\Gamma$ onto $\Gamma_{0}$. Hence, by a theorem of Banach [1, p. 41, Theorem 5] $T$ must be a homeomorphism.

In the case of $\Gamma_{0}=\Gamma$ the foregoing theorem gives a definitive answer to a question left open by Ganapathy Iyer [4, p. 882], concerning the interrelationship between bases in $\Gamma$ and automorphisms on $\Gamma$.

Although proper bases are by definition absolute bases, it is not clear $a$ prior $i$ that every proper basis $\left\{\alpha_{n}\right\}$ is actually a basis. Conceivably $\sum_{n=0}^{\infty} c_{n} \alpha_{n}$ can converge uniformly on compact sets without the same being true of $\sum_{n=0}^{\infty}\left|c_{n} \alpha_{n}\right|$. The following corollary shows that this situation cannot occur when the subspace spanned absolutely by $\left\{\alpha_{n}\right\}$ is closed.

CoRollary 4.1. Let $\left\{\alpha_{n}\right\}$ be a proper basis for a closed subspace $\Gamma_{0}$ of $\Gamma$. Then $\left\{\alpha_{n}\right\}$ is a basis in $\Gamma_{0}$. In fact, if $\left\{c_{n}\right\}$ is any sequence of complex numbers for which $\sum_{n=0}^{\infty} c_{n} \alpha_{n}$ converges uniformly on compact sets, then $\sum_{n=0}^{\infty}\left|c_{n} \alpha_{n}\right|$ converges uniformly on compact sets.

Proof. Suppose that $\sum_{n=0}^{\infty} c_{n} \alpha_{n}$ converges uniformly on compact sets, and let $T$ be a linear homeomorphic mapping of $\Gamma$ onto $\Gamma_{0}$ such that $T \delta_{n}=\alpha_{n}(n=0,1, \cdots)$. Then $T^{-1}\left(\sum_{n=0}^{\infty} c_{n} \alpha_{n}\right)=\sum_{n=0}^{\infty} c_{n} \delta_{n}$ is a function in $\Gamma$, so that $\left|c_{n}\right|^{1 / n} \rightarrow 0$. Since $\left\{\alpha_{n}\right\}$ is a proper basis in $\Gamma_{0}$, this implies that $\sum_{n=0}^{\infty}\left|c_{n} \alpha_{n}\right|$ converges uniformly on compact sets.

Combining mappings in the usual fashion leads to

CoRollary 4.2. If $\left\{\alpha_{n}^{1}\right\}$ and $\left\{\alpha_{n}^{2}\right\}$ are proper bases for closed sub- 
spaces $\Gamma_{1}$ and $\Gamma_{2}$, respectively, of $\Gamma$, then there exists a linear homeomorphic mapping $T$ of $\Gamma_{1}$ onto $\Gamma_{2}$ such that $T \alpha_{n}^{1}=\alpha_{n}^{2}(n=0,1, \cdots)$.

Conversely, let $T$ be a linear homeomorphic mapping of a closed subspace $\Gamma_{1}$ of $\Gamma$ onto a closed subspace $\Gamma_{2}$ of $\Gamma$, and let $\left\{\alpha_{n}^{1}\right\}$ be a proper basis in $\Gamma_{1}$; then the sequence $\left\{\alpha_{n}^{2}\right\}$ defined by $\alpha_{n}^{2}=T \alpha_{n}^{1}(n=0,1, \cdots)$ is a proper basis in $\Gamma_{2}$.

\section{BIBLIOGRAPHY}

1. S. Banach, Theorie des operations linéaires, Warsaw, 1932.

2. V. Ganapathy Iyer, On the space of integral functions. I, J. Indian Math. Soc. (2) vol. 12 (1948) pp. 13-30.

3. - On the space of integral functions. II, Quart. J. Math. Oxford Ser. (2) vol. 1 (1950) pp. 86-96.

4. - On the space of integral functions. III, Proc. Amer. Math. Soc. vol. 3 (1952) pp. 874-883.

5. - On the space of integral functions. IV, Proc. Amer. Math. Soc. vol. 7 (1956) pp. 644-649.

6. S. Karlin, Bases in Banach spaces, Duke Math. J. vol. 15 (1948) pp. 971-985.

UNIVERSITY OF WASHINGTON 